\title{
Use of a Novel Array Detector for the Direct Analysis of Solid Samples by Laser Ablation Inductively Coupled Plasma Sector-Field Mass Spectrometry
}

\author{
James H. Barnes, IV, Gregory D. Schilling, and Gary M. Hieftje \\ Department of Chemistry, Indiana University, Bloomington, Indiana, USA
}

Roger P. Sperline and M. Bonner Denton

Department of Chemistry, University of Arizona, Tucson, Arizona, USA

Charles J. Barinaga and David W. Koppenaal

Pacific Northwest National Laboratory, Richland, Washington, USA

\begin{abstract}
The use of laser ablation (LA) as a sample-introduction method for inductively coupled plasma mass spectrometry (ICP-MS) creates a powerful tool for trace elemental analysis. With this type of instrument, high analyte spatial resolution is possible in three dimensions with $\mathrm{ng} / \mathrm{g}$ limits of detection and minimal sample consumption. Here, simultaneous detection is used to eliminate the correlated noise that plagues the ablation process. This benefit allows analyses to be performed with single laser pulses, resulting in improved depth resolution, even less sample consumption, and improved measurement precision. The new instrument includes an LA sample-introduction system coupled to an ICP ionization source and a Mattauch-Herzog mass spectrograph (MHMS) fitted with a novel array detector. With this instrument, absolute limits of detection are in the tens to hundreds of fg regime and isotope-ratio precision is better than $0.02 \%$ RSD with a one-hour integration period. Finally, depth-profile analysis has been performed with a depth resolution of $5 \mathrm{~nm}$ per ablation event. (J Am Soc Mass Spectrom 2004, 15, 769-776) @ 2004 American Society for Mass Spectrometry
\end{abstract}

$\mathrm{L}$ aser ablation (LA) has become an established sampling method capable of minimally destructive, direct analysis of a variety of sample types [1]. When united with inductively coupled plasma mass spectrometry (ICP-MS), a powerful and sensitive tool for elemental analysis is realized. This is a result of the numerous benefits afforded by both LA sample introduction and ICP-MS. With LA, minimal sample preparation is necessary. Often, a sample can be placed directly in an ablation cell and analyzed. The technique is amenable to numerous sample matrices, including conductive [2] and non-conductive [3] solids, tissue samples [4], liquids [5], and oils [6]. Moreover, with LA it is possible to obtain three dimensions of spatial resolution for analyte concentration [7, 8]. The lateral resolution, though, is limited to single to tens of micrometers by the laser spot size. Other techniques, such as secondary ion mass spectrometry (SIMS), Auger

Published online April 2, 2004

Address reprint requests to Professor G. M. Hieftje, Department of Chemistry, Indiana University, A150 Chemistry Building, 800 East Kirkwood Avenue, Bloomington, IN 47405-7102, USA. E-mail: hieftje@indiana.edu electron spectroscopy (AES), and scanning electron microscopy with energy-dispersive x-ray detection (SEM-EDX) can provide sub-micrometer lateral resolution. Unfortunately, with these techniques, the analysis of large samples can be lengthy and the samples must be placed in vacuum, an inconvenient and time-consuming matter. Moreover, depth profile analysis is not practical with depths over several microns with electron based techniques.

ICP-MS is one of the standard methods for trace elemental analysis. With solution nebulization, sensitivity at or above $10^{9} \mathrm{cps} / \mathrm{ppm}$ is possible, leading to limits of detection in the parts-per-quadrillion (ppq) regime [9]. The annular shape of the ICP allows samples to be introduced axially with little perturbation to the plasma. A final benefit of the ICP is its high gas-kinetic temperature, typically $6000 \mathrm{~K}$, resulting in nearly complete atomization and ionization of all elements.

Though LA-ICP-MS has numerous benefits, the technique suffers from several limitations. The ablation process lacks shot-to-shot precision, resulting in an erratic response and difficulty in quantification [10]. This source of correlated noise results from variations in 
laser power as well as from the interaction of the laser with the sample with each pulse [11-13]. To some extent, this problem can be lessened either through the ratioing of analyte signals to minimize the correlated noise or the averaging effects of high ablation pulse rates and large ablation cells. Unfortunately, the latter solution sacrifices the spatial resolution available with LA. To improve quantification, recent studies have demonstrated that both internal standardization [13] and solution nebulization [14] can be used for calibration.

In addition to the adverse effects of the ablation process on quantification, the lack of reproducibility limits the accuracy and precision of isotope-ratio data. This problem is compounded by the ICP source, which introduces an additional level of multiplicative noise into the measurement. Moreover, large ablated particles, typically over $0.5 \mu \mathrm{m}$ in diameter, are not completely atomized and ionized by the ICP, resulting in elemental and isotopic fractionation [15, 16]. These factors combine to limit isotope-ratio precision for LAICP-MS to values typically around $0.1-1 \%$ RSD for single-channel instruments and $0.006-0.02 \%$ RDS for multicollector instruments [17].

A final problem that plagues analyses by LA, as well as other solid-sampling techniques, is the lack of suitable analytical standards for instrument calibration and quantitative analysis. Since each unique matrix interacts with the laser in a different manner, the amount of ablated material per laser pulse, as well as the particlesize distribution, can vary greatly. Acceptable levels of accuracy are possible only if appropriate matrixmatched standards are available or the aforementioned calibration techniques are utilized.

All of these limitations can be reduced or eliminated through use of simultaneous-extraction instruments, such as time-of-flight (TOF) and ion-trap (IT) mass spectrometers, or by simultaneous-detecting instruments, such as Fourier transform ion cyclotron resonance (FT-ICR) instruments and multiple-collector sector-field (MC-SF) mass spectrometers. Recent studies have demonstrated the ability to perform standardless semi-quantitative analysis with TOFMS $[2,18]$. By monitoring all $\mathrm{m} / \mathrm{z}$ values and normalizing the signal for each $m / z$ to the total ion signal, it is possible to correct for shot-to-shot laser variations. By this method, the total ion current can be related to the ablated mass. With this improvement, similar results can be obtained whether single laser pulses are employed or if the laser is operated at high repetition rates. Moreover, since single laser pulses and fast washout ablation cells can be used with this method, the need for the averaging effects of rapid ablation rates and large-volume ablation cells is eliminated, resulting in improved spatial resolution. Of course, this method obviates the need for matrix-matched standards.

Another benefit of simultaneous detection is that correlated noise can be overcome through ratioing. With correlated noise, such as that introduced by the ablation process and the ICP, all ion signals fluctuate in unison. By ratioing two $\mathrm{m} / \mathrm{z}$ signals, the fluctuations can be reduced or eliminated. Although ratioing can be used with scanning instruments to minimize low-frequency correlated noise, ion signals must be recorded simultaneously to realize the greatest reduction in correlated noise.

A growing field of mass spectrometry research has been the development of SFMS instruments equipped with array detectors capable of acquiring complete mass spectra in a simultaneous manner [19-21]. These instruments take advantage of the benefits of simultaneous detection, such as improved duty cycle, reduced analysis time and sample consumption, and the elimination of correlated noise through ratioing techniques. Moreover, SFMS instruments with array detectors offer an added advantage over other simultaneously detecting instruments, such as TOF or IT mass spectrometers, since they do not need to scan or pulse to obtain a mass spectrum. Additionally, since these arrays can be fabricated with micrometer scale detector elements, both the number of detectors and detector density is greatly increased over what is feasible with MC-SFMS instruments utilizing discrete single-channel detectors, resulting in greater mass coverage. One such array detector, termed the focal plane camera (FPC), has recently been developed and fitted to a Mattauch-Herzog geometry mass spectrograph (MHMS) [21]. A key feature of the MHMS is the presence of a flat focal plane along which all $m / z$ values are focused simultaneously, allowing the use of a planar array detector. This instrument has been coupled with LA sample-introduction and an ICP ionization source. The combination of these techniques creates a powerful tool for solid-sample analysis.

A major goal in the development of the FPC is to improve upon currently available simultaneous-detection instrumentation. Therefore, it is important that the figures of merit for the LA-ICP-MHMS-FPC are comparable to those obtainable with other simultaneousdetection instruments. A great deal of research has been accomplished recently to improve laser ablation performance through coupling to MC-ICP-SFMS. As with other simultaneous detection methods, LA-MC-ICPSFMS instruments realize improved precision through ratioing, offering values better than $0.01 \%$ RSD. Recent studies have shown that the precision can be improved further through mathematical correction methods [22]. A problem that can limit isotope-ratio accuracy during LA sample introduction is isobaric overlaps with the analyte line from polyatomic or multiply-charged species from the matrix material. Waight and co-workers have recently investigated these interferences and the extent to which they limit accuracy [23]. Another important feature of SFMS instruments is high ion transmission, which provides low limits of detection, typically in the single to sub $\mathrm{ng} \mathrm{g}^{-1}$ regime with LA and ICP ionization.

Presented here are the results of coupling LA sample introduction to the ICP-MHMS-FPC. Figures of merit, 
including limits of detection, shot-to-shot precision, and isotope-ratio precision will be detailed. Additionally, the ability of the FPC to monitor transient signals will be discussed. Finally, the utility of the instrument for depth-profile analysis will be explored.

\section{Experimental}

\section{Laser Ablation System}

An LSX-200 laser ablation system (CETAC Technologies, Inc., Omaha, NE) was used in all experimentation. This system utilizes a frequency-quadruple $\mathrm{Nd}$ :YAG laser $(266 \mathrm{~nm})$ to provide laser pulses with energies up to $5 \mathrm{~mJ}$ pulse $^{-1}$. The pulse repetition rate is selectable between 1 and $20 \mathrm{~Hz}$ and the ablation crater can be varied in diameter from 10 to $300 \mu \mathrm{m}$ by means of a ceramic aperture. The ablation cell provided with the system was replaced with one described by Leach and Hieftje [24]. This cell has been designed to provide fast sample washout through use of a low internal volume $\left(11.26 \mathrm{~cm}^{3}\right)$. The ablation volume was swept with helium (0.8 L min ${ }^{-1}$, 99.999, Air Gas, Radnor, PA). Immediately following the ablation cell, an additional flow of argon (0.8 $\mathrm{L} \mathrm{min}^{-1}, 99.999$, Air Gas) was added with a glass $\mathrm{Y}$-connector. This total flow was transferred to the central channel of the ICP torch through $50 \mathrm{~cm}$ of 0.16 $\mathrm{cm}$ i.d. Tygon tubing.

\section{Inductively Coupled Plasma Mass Spectrometer}

An in-house-built Mattauch-Herzog mass spectrograph (MHMS) was used in all experiments [25-27]. This instrument has been coupled to several ionization sources, including both dc and pulsed glow discharges (GD) [21, 28], a microwave plasma torch (MPT) [29], and an ICP [27]. Since the ICP-MHMS has been described previously, only the operating parameters pertinent to the present experiment will be detailed. In addition to the central-channel gas flow from the ablation cell, the ICP was supported with intermediate and outer argon flows of 1.3 and $16 \mathrm{~L} \mathrm{~min}^{-1}$, respectively. The ions were sampled from the ICP at a depth of 12 $\mathrm{mm}$ above the load coil and extracted into vacuum with $0.7,0.7$, and $1.0 \mathrm{~mm}$ sampler, skimmer and third-stage apertures, respectively. The resultant third-stage pressure was $5 \times 10^{-6}$ torr. In all experimentation, the ICP was operated at a forward power of $1.35 \mathrm{~kW}$.

\section{Focal Plane Camera}

The focal plane camera (FPC) has been developed through a collaboration among Indiana University, the University of Arizona, and Pacific Northwest National Laboratory [30]. In its current state of development, the FPC consists of an array of 31 Faraday collectors, spaced $175 \mu \mathrm{m}$ on centers. The active area of each collector is $1.6 \mathrm{~mm}$ tall and $145 \mu \mathrm{m}$ wide, with $10 \mu \mathrm{m}$ ground wires installed between adjacent collectors. The accumulated
Table 1. Operating conditions of the LA-ICP-MHMS-FPC

\begin{tabular}{ll}
\hline Laser Ablation System & \\
Laser repetition frequency & $1-20 \mathrm{~Hz}$ \\
Laser energy & $0-5 \mathrm{~mJ}$ pulse \\
Laser spot diameter & $10-300 \mu \mathrm{m}$ \\
He sweep-gas flow & $0.8 \mathrm{~L} \mathrm{~min}^{-1}$ \\
Ar makeup-gas flow & $0.8 \mathrm{~L} \mathrm{~min}^{-1}$ \\
Inductively Coupled Plasma & \\
Forward power & $1.35 \mathrm{~kW}$ \\
Reflected power & $<5 \mathrm{~W}$ \\
Sampling depth (above load coil) & $12 \mathrm{~mm}$ \\
Outer Ar flow & $16.0 \mathrm{~L} \mathrm{~min}$ \\
Intermediate Ar flow & $1.3 \mathrm{~min}$ \\
Mattauch-Herzog Mass Spectrograph & \\
Sampler aperture diameter & $0.7 \mathrm{~mm}$ \\
Skimmer aperture diameter & $0.7 \mathrm{~mm}$ \\
Third-stage aperture diameter & $1.0 \mathrm{~mm}$ \\
Entrance slit width & $100 \mu \mathrm{m}$ \\
Third-stage pressure & $5 \times 10^{-6}$ torr $^{-1}$ \\
Acceleration potential & $1000 \mathrm{~V}$ \\
Magnetic field strength & $1000-10,000 \mathrm{Gauss}$ \\
lon optic potentials & $\mathrm{Variable}$ \\
Focal Plane Camera & \\
Faraday cup width & $145 \mu \mathrm{m}$ \\
Faraday cup length & $1.6 \mathrm{~mm}$ \\
Faraday cup spacing (on centers) & $175 \mu \mathrm{m}$ \\
Active Faraday cups & 31 \\
Operating temperature & $-40^{\circ} \mathrm{C}$ \\
\hline & \\
&
\end{tabular}

ion signal on each collector is integrated with its own capacitive trans-impedance amplifier; the array of amplifiers is read with a low-noise multiplexer. Further details on the FPC and its coupling to the MHMS can be found elsewhere [21]. The operating conditions of the LA-ICP-MHMS-FPC instrument are summarized in Table 1.

\section{Elemental Analysis Standards}

Both glass and metal standards were used in this study. For measurements of limits of detection, isotope-ratio precision, and laser ablation shot-to-shot reproducibility, a glass standard (NIST 613, Gaithersburg, MD) and several metal standards (BNRM H-1A, H-3A, 44, 88F, 172, Houston, TX) were used. For depth-profile analysis, a copper-on-steel coating-thickness standard (NIST 1359b) was used.

\section{Figure-of-Merit Determination}

Limits of detection (LOD) were calculated both for continuous ablation at $20 \mathrm{~Hz}$ and for single laser ablation pulses using a glass standard (NIST 613) and the BNRM metal standards. For both methods, the laser power was maintained at $5 \mathrm{~mJ}$ pulse ${ }^{-1}$ with a $200 \mu \mathrm{m}$ ablation crater. LOD values were calculated using the following equation [31]:

$$
\operatorname{LOD}_{x}=\frac{3 \sigma[X] n^{1 / 2}}{A}
$$


Table 2. Limits of detection, corrected for isotopic abundance, for several elements with both continues and single-shot laser ablation

\begin{tabular}{lcc}
\hline Element & Steady state $(\mathrm{ng} / \mathrm{g})^{*}$ & Single shot $(\mathrm{fg})$ \\
\hline \hline $\mathrm{B}$ & 31 & 160 \\
$\mathrm{Mn}$ & 51 & 261 \\
$\mathrm{Fe}$ & 36 & 186 \\
$\mathrm{Co}$ & 41 & 210 \\
$\mathrm{Ni}$ & 41 & 216 \\
$\mathrm{Cu}$ & 31 & 163 \\
$\mathrm{Rb}$ & 112 & 577 \\
$\mathrm{Sr}$ & 342 & 1770 \\
$\mathrm{Ag}$ & 15 & 76 \\
$\mathrm{Au}$ & 36 & 188 \\
$\mathrm{Tl}$ & 10 & 50 \\
$\mathrm{~Pb}$ & 9 & 46 \\
$\mathrm{Th}$ & 233 & 1200 \\
$\mathrm{U}$ & 53 & 272 \\
\hline
\end{tabular}

*Laser operated at a pulse-repetition rate of $20 \mathrm{~Hz}$.

where $[X]$ is the concentration of the analyte in the sample in the case of steady-state ablation or the total mass of the analyte ablated for signal-shot ablation, $\sigma$ is the standard deviation of the blank, $A$ is the timeintegrated analyte signal, and $n$ is the number of points used to calculate $\sigma$ and $A$. With steady-state ablation, $A$ was determined for a $10 \mathrm{~s}$ integration while with the single-shot measurements, $A$ is the average signal obtained for 10 laser pulses. Blank levels were determined prior to signal measurement with the LA system off. With these same laser operating conditions, the ability of the instrument to monitor fast transient signals as well as the shot-to-shot precision of the laser-ablation system were determined. The fast-washout ablation cell has been shown to produce transient signals of less than $100 \mathrm{~ms}$ in length [24], requiring the FPC to respond on an even faster timescale.

Depth-profile analysis was performed with a certified standard (NIST 1359b). The NIST standard is comprised of a $45 \mu \mathrm{m}$ layer of copper on a cold-rolled steel substrate. To protect the copper from oxidation, a chromium layer has been electroplated onto the copper surface. Although uncertified, the chromium layer is approximately $1 \mu \mathrm{m}$ thick. For this sample, the laser was initially operated at $20 \mathrm{~Hz}$, with an ablation crater and energy of $100 \mu \mathrm{m}$ and $5 \mathrm{~mJ}$ pulse ${ }^{-1}$, respectively. This method was used to rapidly determine the approximate depth profile of the sample. The laser ablation system was subsequently operated at $2 \mathrm{~Hz}$ with an energy of $1 \mathrm{~mJ}$ pulse $^{-1}$ to obtain a more accurate representation of the depth profile.

\section{Results and Discussion}

\section{Limits of Detection}

LOD values were calculated at a $3 \sigma$ level and are reported in Table 2. These values have been corrected for the natural isotopic abundance of the elements. With the laser ablation system operating in a continuous mode, detection limits are in the tens to hundreds of $\mathrm{ng} / \mathrm{g}$ regime. When single laser pulses are used, absolute detection limits of tens to hundreds of $\mathrm{fg}$ are obtainable. Single-shot values have been determined by calculating the ablated analyte mass based on previous crater measurements in our laboratory [18]. Poorer LOD values are observed for Th and $U$ due to both the instability of the electromagnet at high fields and the penetration of magnetic field lines into the detector region of the instrument. These values are several orders of magnitude poorer than those typically obtained with LA-MC-ICP-SFMS instruments. This discrepancy can be attributed to the mass analyzer, not the FPC detector. Recent studies have shown that comparable limits of detection are obtained with the MHMS using either SEM or FPC detection with both GD and ICP ionization [21, 32]. Moreover, due to the compact nature of the MHMS, a low acceleration potential is utilized $(1 \mathrm{kV})$, resulting in a significant loss in transmission efficiency and sensitivity.

\section{Analysis of Fast Transients}

The transient signal produced by a single laser pulse is shown in Figure 1. The signal obtained for ${ }^{56} \mathrm{Fe}$ has been plotted as a function of time on both a linear (Figure 1a) and logarithmic (Figure 1b) scale for the ablation of BNRM $44(97.87 \% \mathrm{Fe})$. For this study, the bulk material was chosen as the analyte because of a high $\mathrm{S} / \mathrm{N}$ level, allowing the shape of the transient signal to be clearly observed. With the experimental setup used here, transients of $25 \mathrm{~ms}$ (FWHM) are produced. It should be noted that the transient is comprised of two portions, as is apparent from the two linear portions of Figure 1b: An initial, sharp peak in the first $100 \mathrm{~ms}$ caused by the ablation process, and a long, low-intensity tail that persists for several hundred ms as a result of continual washout of ablated particles from the cell and transfer tubing. With the FPC collecting data points every 4.2 $\mathrm{ms}$, both portions of the transient signal are clearly observed. It should be noted that shorter integration periods are not possible with the current device because of the limited time response of the controlling electronics and data acquisition software. Future modifications to the detector will allow improved temporal response.

\section{Precision for Single-Shot Measurements}

It is known that variations in laser power and interaction of the laser with the sample cause fluctuations in LA signal level and limit shot-to-shot precision. With the ability of the FPC to monitor transient signals with millisecond resolution, this process was monitored. Figure 2 shows the ${ }^{56} \mathrm{Fe}$ signal for ten replicate laser ablation pulses applied to sample BNRM 44. A significant variation exists in the peak signal level for the series of ablation events, with a precision of $39 \%$ RSD. If peak area is determined, though, the precision im- 

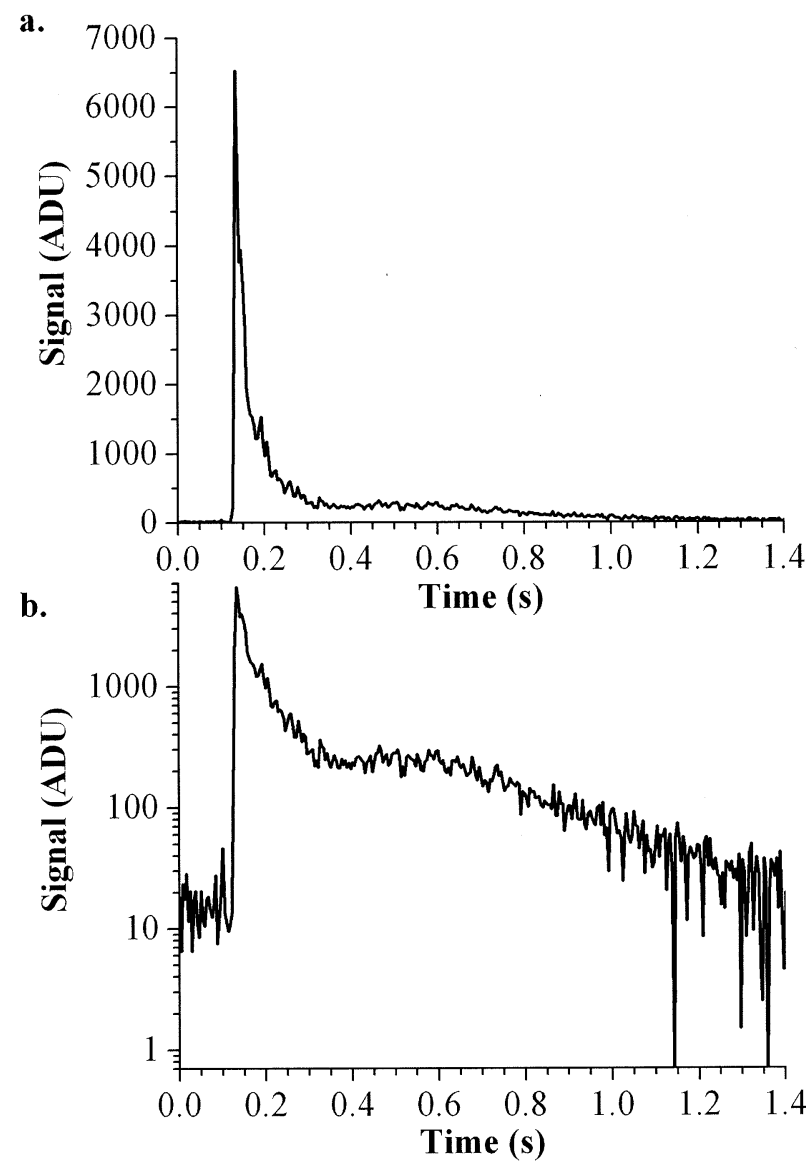

Figure 1. Transient signal for ${ }^{56} \mathrm{Fe}$ produced by a single laser ablation pulse displayed on both linear (a) and logarithmic (b) scales (Energy: $5 \mathrm{~mJ}$ pulse ${ }^{-1}$, spot size: $200 \mu \mathrm{m}$ ). It is clear from (b) that the transient is comprised of a fast portion, with a width of 25 ms FWHM, and a long tail that persists for nearly $1 \mathrm{~s}$.

proves to $2.8 \%$ RSD, suggesting that the ablated mass is relatively consistent. Nevertheless, this source of multiplicative noise will limit the precision of isotope-ratio data unless simultaneous detection is utilized.

\section{Isotope-Ratio Precision}

In previous experiments with solution nebulization, the ICP-MHMS has demonstrated a precision of better than $0.007 \%$ RSD with FPC detection [32] and values near $0.5 \%$ RSD with single channel detection [27]. To determine the precision of the instrument with LA sample introduction, the laser was operated at $20 \mathrm{~Hz}$ with an energy of $5 \mathrm{~mJ}$ pulse $^{-1}$. The isotopes of molybdenum were monitored for sample BNRM 44 and the precision calculated from ${ }^{95} \mathrm{Mo}$ and ${ }^{96} \mathrm{Mo}(0.51 \%)$. A plot of the signal for these two isotopes for a $100 \mathrm{~s}$ ablation period is shown in Figure 3. Additionally, on this plot is shown the ratio of the two $\mathrm{m} / \mathrm{z}$ signals. From this plot, the multiplicative noise introduced by the ablation process is clearly visible. Since the noise is correlated, the signals for the two isotopes fluctuate in a similar manner. Even though signals from the individual iso-

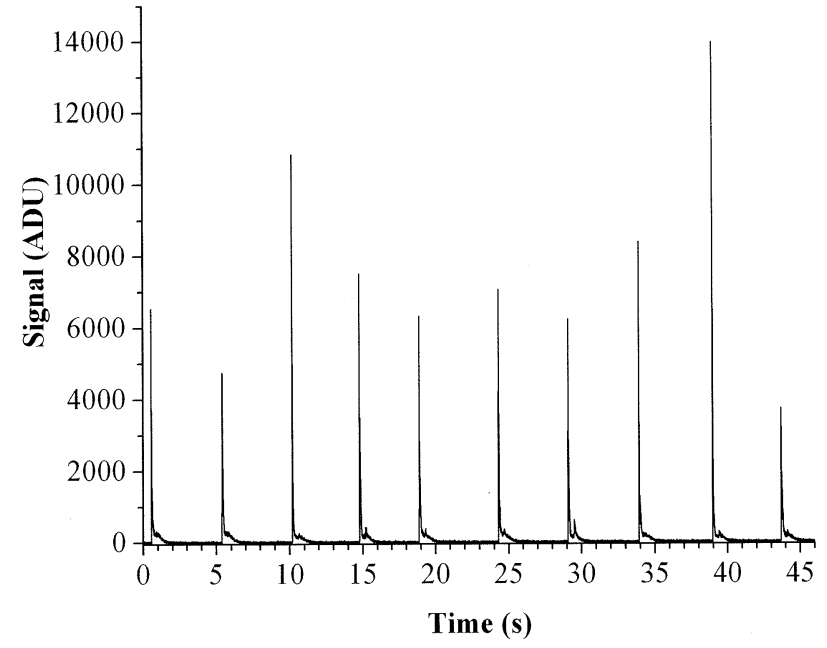

Figure 2. Transient signals produced by the LA-ICP-MHMS-FPC for ten replicate laser ablation pulses (Energy: $5 \mathrm{~mJ}$ pulse $^{-1}$, spot size: $200 \mu \mathrm{m})$. From this plot, the imprecision of the ablation process is apparent.

topes exhibit a precision of poorer than $15 \%$ RSD, the ratio precision is better than $1 \%$ RSD. This ratio precision can be improved even further with longer integration periods. It was experimentally determined that with a one-h integration time, consisting of $106-\mathrm{min}$ integration periods, a precision of $0.018 \%$ RSD is obtainable. This value is comparable to that obtained with other LA-ICP-MS multicollector instruments and better than that obtainable with single channel instruments [17]. Furthermore, when this value is compared with the precision obtainable with solution nebulization on this same instrument $(0.007 \%)$, it is clear that the laser ablation process added some uncertainty. Still, the

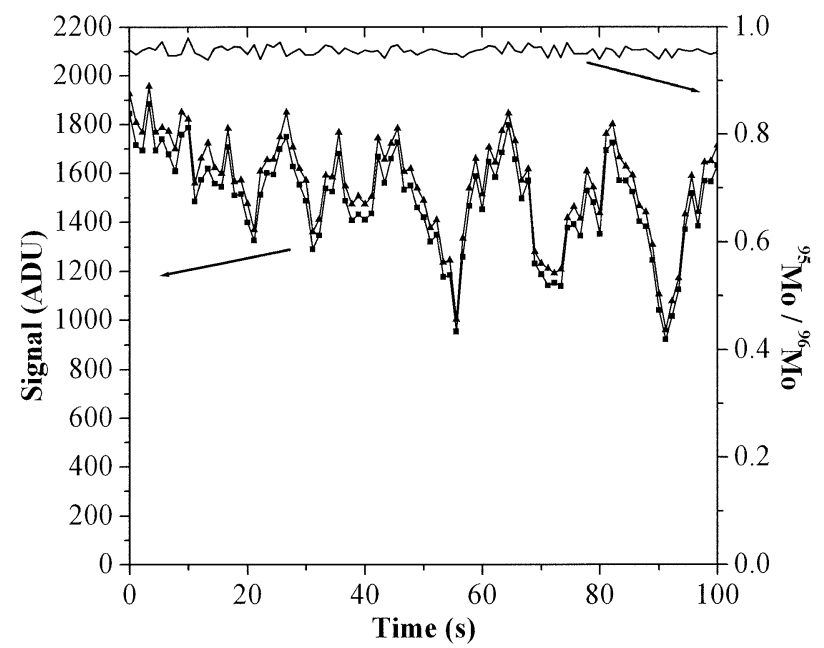

Figure 3. Although the ablation process is plagued with multiplicative noise, as seen with the signal for ${ }^{95} \mathrm{Mo}$ (filled square) and ${ }^{96} \mathrm{Mo}$ (filled triangle), this noise can be reduced through rationing techniques (solid line). The individual $\mathrm{m} / \mathrm{z}$ signals exhibit a precision of poorer than $15 \%$ RSD while the ratio precision improves to better than $1 \%$ RSD. 


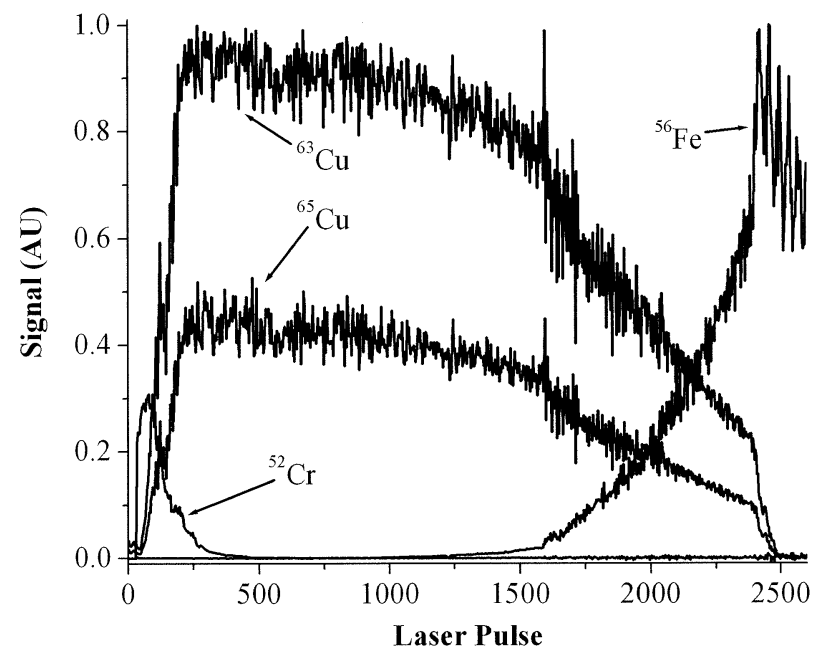

Figure 4. Depth profile analysis of sample NIST 1359b. The three distinct layers of the standard are visible: the $1 \mu \mathrm{m}$ chromium protective layer, the $45 \mu \mathrm{m}$ copper layer, and the cold rolled steel substrate. With the LA system operating at rapid ablation rates (20 $\mathrm{Hz}$ ) and high energy (5 mJ pulse $\left.{ }^{-1}\right)$, a depth resolution of $30 \mathrm{~nm}$ pulse $^{-1}$ is realized.

precision is significantly improved over that obtainable with the MHMS and a single-channel detector. It should be noted that the precision has reached the countingstatistics limit, and cannot be further improved with longer integration times.

It is important to note several factors that serve to limit the precision obtainable with this instrument. The MHMS has been fabricated in-house and is therefore more prone to noise sources than are some commercial MC-SFMS instruments. Such noise sources include RF interference from the ICP, plasma flicker, temperature fluctuations, mechanical pump noise, and magnet noise and drift, all of which require long integration periods to reduce. The latter noise source, from the magnet, degrades precision the most, since it can cause a timedependent fluctuation in isotope-ratio values. Minimizing or eliminating this noise source should result in improved precision and reduced integration time.

\section{Depth Profile Analysis}

One of the key benefits of LA is the ability to obtain three spatial dimensions of concentration information, both laterally along the surface of the sample and through depth profiling. The benefit of LA for depth profiling was demonstrated by means of the NIST $1359 \mathrm{~b}$ standard. With rapid ablation rates $(20 \mathrm{~Hz})$ and high laser energy (5 $\mathrm{mJ}$ pulse ${ }^{-1}$ ), the rough depth profile can be seen in Figure 4. The signal levels in Figure 4 have all been normalized to the most intense $\mathrm{m} / \mathrm{z}\left({ }^{63} \mathrm{Cu}\right)$; also, the data were collected in two mass windows: from $m / z=52$ to $m / z=59$ and from $m / z=59$ to $m / z=65$. Additionally, because the transition between layers is not instantaneous due to effects such as non-flat-top crater profiles and edge effects, the point

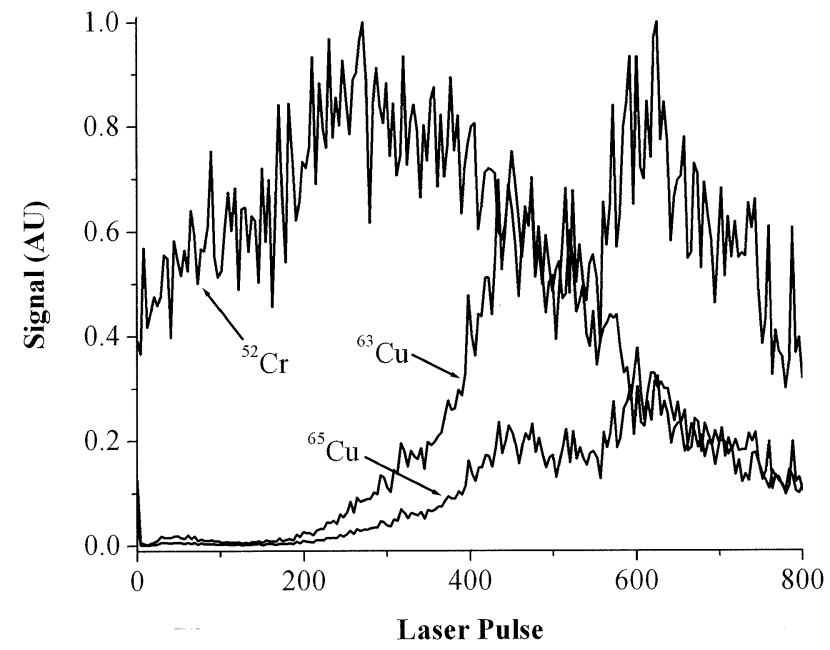

Figure 5. To improve the depth resolution, the LA system was operated at a slower ablation rate $(2 \mathrm{~Hz})$ and lower laser energy $(1$ $\mathrm{mJ}$ pulse $\left.{ }^{-1}\right)$. Under these conditions, the $1 \mu \mathrm{m}$ chromium protective layer was analyzed with a depth resolution of $5 \mathrm{~nm}$ pulse $^{-1}$.

where the signal from the underlying layer was first observed was used to indicate the transition point between layers. Under these operating conditions, it takes approximately 1500 laser pulses to ablate through the copper layer and into the cold-rolled steel substrate. With a copper-layer thickness of $45 \mu \mathrm{m}$, this corresponds to a depth per pulse of $30 \mathrm{~nm}$.

To further examine the depth-resolution capabilities of the LA system, the laser energy was reduced to $1 \mathrm{~mJ}$ pulse $^{-1}$ and the ablation rate was slowed to $2 \mathrm{~Hz}$. Under these conditions, only the chromium protective layer of NIST $1359 \mathrm{~b}$ was examined. The depth profile for these operating conditions can be found in Figure 5. Under these conditions, it takes approximately 200 laser pulses to ablate through the chromium layer and reach the copper. With a chromium layer thickness of $1 \mu \mathrm{m}$, this corresponds to a depth per pulse of $5 \mathrm{~nm}$. This value would represent the depth resolution, assuming a constant ablation rate, vertical-walled ablation craters, and flat crater bottoms. Because none of these requirements is satisfied, the depth resolution is somewhat worse.

\section{Conclusions}

Laser ablation sample introduction has been successfully coupled to an inductively coupled plasma ionization Mattauch-Herzog mass spectrograph equipped with a novel detector array. With this instrument, limits of detection in the tens to hundreds of $\mathrm{ng} / \mathrm{g}$ regime are obtainable with continuous ablation while values in the tens to hundreds of $\mathrm{fg}$ range are realized with single laser ablation pulses. The FPC demonstrated the ability to monitor transient signals of tens of milliseconds in duration through use of a fast-washout ablation cell. Although somewhat modest conditions have been used in this study, the simultaneous detection ability of the 
instrument has demonstrated that the correlated noise associated with the ablation process can be reduced through ratioing techniques, leading to an isotope-ratio precision of better than $0.02 \%$ RSD. A final benefit realized with this coupling is the ability to perform depth profile analyses, with depth resolution as high as $5 \mathrm{~nm}$ per laser pulse.

The coupling of LA to ICP-MS has proven to be a powerful tool for the direct analysis of solid samples. Single-channel instruments provide in many cases limits of detection in the single $\mathrm{ng} \mathrm{g}^{-1}$ regime and precision better than $0.1 \%$ RSD. These figures of merit are further improved with MC-SFMS instruments. With simultaneous multielement, multi-isotope detection, analyses can be completed more rapidly, resulting in smaller required sample sizes and better absolute detection limits. Additionally, correlated noise sources can be compensated through ratioing, allowing measurement precision to approach $0.001 \%$ RSD. Although these instruments perform quite well, room for improvement exists. With commercial MC-SFMS instruments, only between 10 and $20 \mathrm{~m} / z$ values can be simultaneously recorded. Although this number might seem adequate, it is near the upper limit of detectable masses, since little room exists in these instruments for additional detectors. Moreover, these instruments cover only a small mass window so, for example, it is not possible to simultaneously detect elements such as strontium and uranium.

Though not possible with the present generation of cameras, the FPC has been designed to address the aforementioned limitations. Planned revisions of this technology will allow complete atomic mass spectra to be acquired continuously and simultaneously. With this technology, the full benefit of simultaneous detection can be realized.

\section{Acknowledgments}

Support for this work was provided by the U.S. Department of Energy, Office of Nonproliferation Research and Engineering. Pacific Northwest National Laboratory is operated by Battelle Memorial Institute for the Department of Energy under contract DE-AC06-76RLO-1830. The authors are grateful to CETAC Technologies, Inc. for use of the laser ablation system described in this study.

\section{References}

1. Günther, D.; Horn, I.; Hattendorf, B. Recent Trends and Developments in Laser Ablation-ICP-Mass Spectrometry. Fresenius J. Anal. Chem. 2000, 368, 4-14.

2. Leach, A. M.; Hieftje, G. M. Identification of Alloys Using Single Shot Laser Ablation Inductively Coupled Plasma Timeof-Flight Mass Spectrometry. J. Anal. At. Spectrom. 2002, 17, 852-857.

3. Lee, Y.-L.; Chang, C.-C.; Jiang, S.-J. Laser Ablation Inductively Coupled Plasma Mass Spectrometry for the Determination of Trace Elements in Soil. Spectrochim. Acta, Part B 2003, 58B(523), 530.
4. Ghazi, A. M.; Wataha, J. C.; O’Dell, N. L.; Singh, B. B.; Simmons, R.; Shuttleworth, S. Quantitative Concentration Profiling of Nickel in Tissues Around Metal Implants: a New Biomedical Application of Laser Ablation Sector Field ICPMS. J. Anal. At. Spectrom. 2002, 17, 1295-1299.

5. Günther, D.; Frischknecht, R.; Muschenborn, H. J.; Heinrich, C. A. Direct Liquid Ablation. A New Calibration Strategy for Laser Ablation-ICP-MS Microanalysis of Solids and Liquids. Fresenius J. Anal. Chem. 1997, 359, 390-393.

6. Bings, N. H. Direct Determination of Metals in Lubricating Oils by Laser Ablation Coupled to Inductively Coupled Plasma Time-of-Flight Mass Spectrometry. J. Anal. At. Spectrom. 2002, 17, 759-767.

7. Bleiner, D.; Plotnikov, A.; Vogt, C.; Wetzig, K.; Günther, D. Depth Profile Analysis of Various Titanium Based Coatings on Steel and Tungsten Carbide Using Laser Ablation Inductively Coupled Plasma Time of Flight Mass Spectrometry. Fresenius J. Anal. Chem. 2000, 368, 221-226.

8. Plotnikov, A.; Vogt, C.; Hoffmann, V.; Taeschner, C.; Wetzig, K. Application of Laser Ablation Inductively Coupled Plasma Quadrupole Mass Spectrometry (LA-ICP-QMS) for Depth Profile Analysis. J. Anal. At. Spectrom. 2001, 16, 1290-1295.

9. Becker, J. S.; Dietze, H.-J. Inorganic Mass Spectrometric Methods for Trace, Ultratrace, Isotope, and Surface Analysis. Int. J. Mass Spectrom. 2000, 197, 1-35.

10. Russo, R. E. Laser Ablation. Appl. Spectrosc. 1995, 49, 14A-28A.

11. Rodushkin, I.; Axelsson, M. D.; Malinovsky, D.; Baxter, D. C. Analyte- and Matrix-Dependent Elemental Response Variations in Laser Ablation Inductively Coupled Plasma Mass Spectrometry. Part 2. Implications for Multi-Element Analyses. J. Anal. At. Spectrom. 2002, 17, 1231-1239.

12. Rodushkin, I.; Axelsson, M. D.; Malinovsky, D.; Baxter, D. C. Analyte- and Matrix-Dependent Elemental Response Variations in Laser Ablation Inductively Coupled Plasma Mass Spectrometry. Part 1. The Roles of Plasma and Ion Sampling Conditions. J. Anal. At. Spectrom. 2002, 17, 1223-1230.

13. Guillong, M.; Horn, I.; Günther, D. Capabilities of a Homogenized $266 \mathrm{~nm}$ Nd: YAG Laser Ablation System for LA-ICPMS. J. Anal. At. Spectrom. 2002, 17, 8-14.

14. Leach, J. J.; Allen, L. A.; Aeschliman, D. B.; Houk, R. S. Calibration of Laser Ablation Inductively Coupled Plasma Mass Spectrometry Using Standard Additions with Dried Solution Aerosols. Anal. Chem. 1999, 71, 440-445.

15. Guillong, M.; Kuhn, H.-R.; Günther, D. Application of a Particle Separation Device to Reduce Inductively Coupled Plasma-Enhanced Elemental Fractionation in Laser Ablation Inductively Coupled Plasma Mass Spectrometry. Spectrochim. Acta Part B 2003, 58B(211), 220.

16. Guillong, M.; Günther, D. Effect of Particle Size Distribution on ICP-Induced Elemental Fractionation in Laser AblationInductively Coupled Plasma-Mass Spectrometry. J. Anal. At. Spectrom. 2002, 17, 831-837.

17. Becker, J. S. State-of-the-Art and Progress in Precise and Accurate Isotope-Ratio Measurements by ICP-MS and LAICP-MS. J. Anal. At. Spectrom. 2002, 17, 1172-1185.

18. Leach, A. M.; Hieftje, G. M. Standardless Semiquantitative Analysis of Metals Using Single-Shot Laser Ablation Inductively Coupled Plasma Time-of-Flight Mass Spectrometry. Anal. Chem. 2001, 73, 2959-2967.

19. Darling, R. B.; Scheidemann, A. A.; Bhat, K. N.; Chen, T. C. Micromachined Faraday Cup Array Using Deep Reactive Ion Etching. Sens. Act. A 2002, A95, 84-93.

20. Fuerstenau, S.; Soli, G.; Cunningham, T.; Hancock, B.; Pain, B.; Sinha, M. Active Pixel Sensors for Mass Spectrometry. Int. J. Mass Spectrom. 2002, 215, 101-111.

21. Barnes, IV, J. H.; Sperline, R.; Denton, M. B.; Barinaga, C. J.; Koppenaal, D.; Young, E. T.; Hieftje, G. M. Characterization of 
a Focal Plane Camera Fitted to a Mattauch-Herzog Geometry Mass Spectrograph. 1. Use with a Glow-Discharge Source. Anal. Chem. 2002, 74, 5327-5332.

22. Hirata, T.; Hayano, Y.; Ohno, T. Improvements in Precision of Isotopic Ratio Measurements Using Laser Ablation-Multiple Collector-ICP-Mass Spectrometry: Reduction of Changes in Measured Isotopic Ratios. J. Anal. At. Spectrom 2003, 18, 1283-1288.

23. Waight, T.; Baker, J.; Peate, D. Sr Isotope-Ratio Measurements by Double-Focusing MC-ICPMS: Techniques, Observations and Pitfalls. Int. J. Mass Spectrom. 2002, 221, 229-244.

24. Leach, A. M.; Hieftje, G. M. Factors Affecting the Production of Fast Transient Signals in Single Shot Laser Ablation Inductively Coupled Plasma Mass Spectrometry. Appl. Spectrosc. 2002, 56, 62-69.

25. Burgoyne, T. W.; Hieftje, G. M.; Hites, R. A. Design and Performance of a Plasma-Source Mass Spectrograph. J. Am. Soc. Mass Spectrom. 1997, 8, 307-318.

26. Burgoyne, T. W.; Hieftje, G. M.; Hites, R. A. Space Charge Evaluation in a Plasma-Source Mass Spectrograph. Anal. Chem. 1997, 69, 485-489.

27. Solyom, D. A.; Gron, O. A.; Barnes, IV, J. H.; Hieftje, G. M. Analytical Capabilities of an Inductively Coupled Plasma
Mattauch-Herzog Mass Spectrometer. Spectrochim. Acta Part B 2001, 56B, 1717-1729.

28. Solyom, D. A.; Hieftje, G. M. Advancing the Capabilities of a Glow-Discharge Sector-Field Mass Spectrometer. J. Anal. At. Spectrom. 2002, 17, 329-333.

29. Barnes, IV, J. H.; Gron, O. A.; Hieftje, G. M. Characterization of an Argon Microwave Plasma Torch Coupled to a MattauchHerzog Geometry Mass Spectrometer. J. Anal. At. Spectrom. 2002, 27, 1132-1136.

30. Knight, A. K.; Sperline, R. P.; Hieftje, G. M.; Young, E.; Barinaga, C. J.; Koppenaal, D. W.; Denton, M. B. The Development of a Micro-Faraday Array for Ion Detection. Int. J. Mass Spectrom. 2002, 215, 131-139.

31. Brushwyler, K. R.; Furuta, N.; Hieftje, G. M. Use of a Spectrally Segmented Photodiode-Array Spectrometer for Inductively Coupled Plasma Atomic Emission Spectroscopy. Examination of Procedures for the Evaluation of Detection Limits. Talanta 1990, 37, 23-32.

32. Barnes, IV, J. H.; Schilling, G. D.; Sperline, R.; Denton, M. B.; Young, E. T.; Barinaga, C. J.; Koppenaal, D. W.; Hieftje, G. M. Characterization of a Focal Plane Camera Fitted to a MattauchHerzog Geometry Mass Spectrograph. 2. Use with an Inductively Coupled Plasma, submitted. 